\title{
La colaboración periodística en Internet y el caso de 'The Stream' de Al Jazeera
}

\author{
Cristina RENEDO FARPÓN \\ Universitat Oberta de Catalunya \\ renedof.cristina@gmail.com
}

\section{Resumen:}

El uso de las nuevas tecnologías ha cambiado la forma de consumir y producir información. La producción de contenidos se ha democratizado y se han abierto canales que fomentan la participación ciudadana, incluso en los grandes medios de comunicación. Las plataformas de redes sociales también son protagonistas de esta tendencia, como presenta el caso del programa The Stream, de Al Jazeera, que mezcla la información de fuentes ciudadanas en plataformas digitales con el tratamiento periodístico.

Palabras Clave:_Periodismo colaborativo; redes sociales; Internet; comunicación 2.0; comunicación digital.

\section{The journalistic collaboration in Internet and the case of 'The Stream' Al Jazeera}

\begin{abstract}
:
The use of new technologies has changed the way people consume and produce information. Internet has democratized content production and created new channels for citizen participation, including major media incorporate these channels to its users. The social networks are also players of this trend. An example of this involvement is The Stream program is aired on Al Jazeera. The program combines public information on digital platforms and professional journalistic treatment of the issues.
\end{abstract}

Key Words: Journalistic collaboration; social networks; Internet; digital communication; web 2.0

\section{Referencia normalizada:}

Renedo Farpón, C. (2014): La colaboración periodística en Internet y el caso de "The Stream" de Al Jazeera. Historia y Comunicación Social. Vol. 19. Núm. Especial Enero. Págs. 613-621.

Sumario: 1. Introducción 2. Metodología 3. Herramientas para un periodismo colaborativo 3.1. Colaboración entre usuarios 3.2. Colaboración entre usuarios y profesionales 4. El caso de The Stream 4.1. 'The Stream' como ejemplo de periodismo colaborativo en la era digital 5. Conclusiones 


\section{Introducción}

El uso de Internet y de las nuevas tecnologías, que se ha ampliado masivamente en la sociedad en los últimos años, ha modificado la forma de acceso y consumo de información por parte de los usuarios. Además, las nuevas tecnologías también han conseguido democratizar la producción de contenidos y, en la actualidad, cualquier persona conectada a la Red y con unos conocimientos básicos de informática es capaz de producir su propia información a través de herramientas tan diferentes como las páginas web, las redes sociales, los wikis, las bitácoras, las plataformas de vídeo, de fotografía o los foros online.

Esta tendencia no ha pasado desapercibida para los grandes medios de comunicación tradicionales, que en sus ediciones digitales han abierto canales de participación en los que ofrecen a los usuarios la posibilidad de subir sus propios contenidos, para convertirles de esta manera en prosumidores, o les da la opción de interactuar con la información, el medio de comunicación u otros lectores a través de herramientas como las redes sociales, los foros, los comentarios en las noticias o los sistemas de votación de contenidos.

Pero en esta era digital la participación colaborativa entre profesionales y usuarios para construir información periodística ha dado lugar a nuevas formas de producción y nuevos modelos de negocio. Los ejemplos van desde el uso de crowdfunding para financiar proyectos periodísticos realizados por profesionales, en los que los usuarios deciden qué productos quieren apoyar económicamente, hasta el mediático caso de Wikileaks, en el que la filtración de documentos de interés público se llevó a cabo gracias en gran parte a informadores anónimos.

En este artículo se van a estudiar estas nuevas formas de producción y, en concreto, se va a analizar el caso del programa 'The Stream', de Al Jazeera, un formato que cuenta además con una plataforma online que permanece activa las 24 horas del día. 'The Stream' aúna la información proporcionada por los ciudadanos en la Red a través de herramientas como las redes sociales, los blogs o las plataformas de vídeo, con el trabajo de periodistas profesionales encargados de contrastar y contextualizar la información, así como de filtrar la más relevante o la que presenta un mayor interés público y periodístico. Una evidencia de las posibilidades del periodismo colaborativo en la era digital.

\section{Metodología}

El presente artículo se centra en el estudio de la construcción de productos periodísticos de manera colaborativa en entornos digitales, y presta especial atención al caso del programa 'The Stream' de Al Jazeera. Para desarrollar el estudio se ha optado por una metodología multidisciplinar con la combinación de las técnicas de investigación cuantitativa y cualitativa. 
En la primera parte del estudio se ha realizado un análisis bibliográfico sobre el periodismo colaborativo en Internet, con el objetivo de definir de manera adecuada el concepto y establecer un marco teórico en la investigación. Se han utilizado tanto estudios y ensayos teóricos sobre la noción de periodismo colaborativo como análisis de ejemplos concretos que aúnan el trabajo de periodistas profesionales con la información producida por usuarios para conocer mejor las herramientas disponibles en la actualidad para desarrollar este tipo de contenidos, así como sus efectos dentro de la audiencia y los usuarios activos.

Además, en el estudio bibliográfico también se ha buscado contextualizar el nacimiento del programa 'The Stream' así como la evolución y situación actual de la cande $\mathrm{Al}$ Jazeera, para comprender mejor la dinámica del programa, relacionada con la denominada 'Primavera árabe'.

En cuanto al análisis metodológico, se ha llevado a cabo un estudio en el que se han utilizado técnicas como la observación, obtención de datos y el análisis, tanto de los contenidos del programa The Stream como de las historias vertidas en su página web, así como la participación de los usuarios, los tipos de canales utilizados y las temáticas más comunes en el programa. Además se ha realizado un análisis comparativo del programa con otros modelos de periodismo participativo existentes en la Red.

\section{Herramientas para un periodismo colaborativo}

La evolución de las nuevas tecnologías ha provocado una acelerada transformación en los medios de comunicación, especialmente en entornos digitales. Los contenidos en Internet, que han crecido de manera vertiginosa gracias al aumento del número de usuarios de la Red, especialmente en los primeros años del siglo XXI, ya no se ven limitados por las barreras espaciotemporales que caracterizaban a los medios de comunicación tradicionales. Esto ha provocado que los consumidores tengan un acceso casi ilimitado a una gran cantidad de información de manera inmediata e independientemente de su localización. Pero no solo han variado las formas de consumo de información por parte de los usuarios, la producción de contenidos también se ha visto afectada por el uso de las herramientas digitales y en la actualidad cualquier persona con conexión a Internet y unas básicas nociones de informática y navegación es capaz de crear y compartir sus propios contenidos.

Castells (2004) ejemplifica esto en su definición de una sociedad en Red en la que prevalece la comunicación en entornos multimedia, dirigida a una audiencia de masas y con un predominio de mensajes bidireccionales, donde los medios presentan un alto nivel de interactividad en su contenido y pueden ser replicados por los consumidores e interlocutores. Además, se han eliminado barreras espaciales y los consumidores pueden tener acceso a través de sus conexiones a todo tipo de información publicada en cualquier lugar del planeta. 
La aparición de herramientas como wikis, bitácoras o blogs, páginas para alojar contenido multimedia o plataformas de redes sociales, que facilitan la conversación entre usuarios y la difusión de contenidos, han favorecido la producción por parte de los usuarios, que ya no se limitan a ser consumidores pasivos de la información de los medios y reclaman una mayor participación. Esta democratización en la producción de la información ha generado un nuevo espacio comunicativo en el que el debate global es posible y han surgido nuevos términos como periodismo colaborativo o periodismo ciudadano, que Howard Rheingold (2011): define como "un cambio en las instituciones profesionales y en la propia naturaleza de la democracia". Estos conceptos han estrechado la relación entre periodistas y usuarios, que ahora pueden colaborar en la construcción de la información de una manera más inmediata y directa.

Como indica Pisani (2006) "la participación ciudadana no es más que una ampliación de las relaciones existentes desde los orígenes entre cualquier medio de comunicación y su audiencia", por lo que no nos encontramos ante algo nuevo ni exclusivo de los medios digitales. Antes de la irrupción de Internet, los usuarios contaban con espacios de participación reducidos y muy moderados por los medios, como las cartas al director en los diarios o la participación telefónica en la radio y televisión, pero Internet ha multiplicado la interactividad entre consumidores y medios de comunicación a través de herramientas como los foros, los comentarios en las noticias, la valoración de artículos o la difusión de noticias a través de redes sociales. Además, la evolución tecnológica y las necesidades de los usuarios han provocado que la colaboración ciudadana para participar en la producción de información se amplíe mucho más allá de los espacios delimitados por los propios medios de comunicación. En este sentido, han surgido en Internet movimientos de colaboración, tanto entre los propios usuarios como con profesionales de la información. De acuerdo con Shayne Bowman y Chris Willis (2013), "este periodismo participativo define el acto de un ciudadano, o un grupo de ciudadanos, con un rol activo en el proceso de recogida, análisis y difusión de noticias e información".

\subsection{Colaboración entre usuarios}

En Internet la colaboración en la producción de contenidos no es algo reciente. Como indica Abbate (2000) "en Arpanet apenas existía distinción entre productores y usuarios y su desarrollo se llevó a cabo gracias a la flexibilidad y colaboración de técnicos y a las prácticas y decisiones sociales de usuarios no expertos que definieron su uso y características actuales". El código abierto ha sido una de las claves en la evolución de este mediot y ha posibilitado su desarrollo así como la creación de un espíritu de colaboración digital. Según Scolari (2010: 191) este sistema "se presenta como un caso extremo dentro de la economía del conocimiento y abre nuevas perspectivas a los investigadores de la cooperación a gran escala”.

Uno de los casos más notables de colaboración entre usuarios aplicando esta filosofía es el de Wikipedia, una enciclopedia libre, editada de forma colaborativa por usuarios de todas pates del mundo, abierta y que se ha situado como una de las páginas web con más referencias de Internet. Su éxito ha propiciado la aparición de 
otros proyectos surgidos dentro de la misma fundación, como es el caso del portal Wikinoticias, una fuente de noticias de contenido libre que funciona de forma muy similar a su predecesora. Este portal basa sus contenidos en la redacción desde un punto de vista neutral y la verificabilidad de sus noticias a través de un sistema de referencias. El portal cuenta además con una revisión de los artículos por parte de editores de prensa, usuarios experimentados del proyecto que, si bien no tienen por qué ser profesionales de la comunicación, muestran con su actividad esa tendencia de cooperación en la creación de información entre productores y usuarios. Otro ejemplo de colaboración entre usuarios, alejado de la cultura Wiki, es el del movimiento bloguero. Como señala Scolari (2010: 196), los usuarios de estas herramientas utilizan "intercambios de listas de weblogs (blogrolls), dando lugar a un proceso de agregación y creación de comunidades". De esta manera los blogs con contenidos temáticos similares se enriquecen mutuamente a través de intercambios de enlaces que enaltecen su contenido y posicionamiento web.

En definitiva, existen numerosas plataformas en las que sus usuarios, formando una comunidad virtual, crean y comparten contenidos: plataformas de fotografías y vídeo (Flickr, Youtube o Vimeo) foros temáticos, comunidades surgidas a través de juegos online y sitios de realidad virtual (Minecraft, Secondlife), plataformas de redes sociales (Twitter, Facebook) o los agregadores de noticias (Digg, Menéame) son ejemplos de este predominio de colaboración que caracteriza la web.

\subsection{Colaboración entre usuarios y profesionales}

Además de la producción de información por parte exclusiva de usuarios, también han surgido un gran número de proyectos en los que la colaboración de ciudadanos como productores de información con periodistas y técnicos profesionales da lugar a un nuevo producto informativo. Las nuevas tecnologías, que facilitan la publicación y el acceso instantáneo a un gran tipo de contenidos escritos y audiovisuales, convierten a los usuarios en fuentes de información para los periodistas profesionales, que ejercen una labor de contrastación, verificación y tratamiento de la información que sea de mayor interés para ofrecer el producto final a los consumidores.

\section{El caso de The Stream}

El programa 'The Stream' de Al Jazeera es un ejemplo de los productos informativos que veíamos en el anterior apartado en los que el periodista colabora con el usuario para construir contenidos a través del aprovechamiento de herramientas digitales. Pero antes de adentrarse en el análisis del programa 'The Stream' y de la plataforma online que lo integra, es oportuno reflexionar sobre el contexto social que se ha vivido durante su nacimiento, así como la situación de la cadena que lo emite, la versión inglesa de Al Jazeera. 
La emisora, que fue fundada en 1996 por el gobierno de Catar, aunque más adelante consiguió su independencia económica a través de la venta de sus propios productos. Su fama comenzó a asentarse en Estados Unidos a partir de los atentados del 11 de septiembre y las posteriores emisiones en exclusiva del conflicto de Afganistán. Sin embargo, su popularidad más reciente a nivel internacional se ha debido a sus emisiones en la emisora inglesa de la cadena relacionadas con la denominada "primavera árabe".

Esta revolución, como indica Kristina Kaush (2001: 24-31), enmarca "las revoluciones en Túnez y Egipto y las subsecuentes protestas en Yemen, Jordania, Argelia y otros países de la región que han dado lugar a grandes expectativas sobre un "efecto dominó": un cambio sísmico que haría que el mundo árabe se tornase finalmente democrático." Además, en estos conflictos, el papel de las nuevas tecnologías, y especialmente del uso ciudadano de las redes sociales, blogs y plataformas de vídeo, se ha caracterizado como algo fundamental en su desarrollo. Como señala Yves González Quijano (2011) "todo comentario sobre la «primavera árabe» implica que la palabra «revolución» esté acompañada de términos como «Facebook» o «Twitter» y más que su «carácter árabe», en definitiva el acontecimiento parece constituirlo el hecho de que estas «revoluciones 2.0» inaugurarían una nueva era en la cual el uso de las redes sociales da una dimensión inédita a la política". Durante el año 2011 los medios de comunicación destacaban el uso de los canales sociales digitales por parte de los ciudadanos para difundir contenidos alternativos a los que emitían los medios oficiales y generalistas.

Pero las redes sociales y la participación ciudadana no han sido las única claves en de la comunicación de estas revueltas, Yves Gonzalez (2011) reflexiona también sobre cómo este efecto de "las redes sociales-con impactos muy variables según los países- conviven hoy con cadenas como Al-Jazeera, lo que genera un nuevo «ecosistema mediático» que contribuyó a construir y difundir el gran relato colectivo de la revolución democrática árabe.". Además, "La mayoría de los testimonios sobre los acontecimientos que han marcado la actualidad del mundo árabe insisten en el considerable rol desempeñado por las cadenas satelitales de la región, sobre todo Al-Jazeera". Este papel de la cadena en las revoluciones árabes también es destacado por José Abu-Tarbush (2011) que define la emisora como "un eficaz medio de socialización política, más apegado a la realidad social árabe, con mayor credibilidad y receptividad del público en general y de las nuevas generaciones en particular. En definitiva, ha logrado transmitir la información sobre los árabes vista por los propios árabes, propiciando una mayor sensibilización y concienciación política acerca de su entorno social, político, económico y regional en contraste con las tendencias existentes en el sistema internacional". Y precisamente es en este marco de comunicación convergente entre el trabajo de Al-Jazeera y la difusión ciudadana de las revueltas árabes a través de las redes sociales, donde nació el programa 'The Stream', el 18 de abril de 2011, fecha en la que también se publicó su plataforma online.

4.1. "The Stream" como ejemplo de periodismo colaborativo en la era digital 
El programa televisivo 'The Stream' así como toda la actividad en torno a su página web, supone un formato más del periodismo colaborativo que se definía en anteriores apartados. Sus contenidos se basan en la participación ciudadana a través de las herramientas que facilitan las nuevas tecnologías, consideradas clave dentro de los conflictos de la 'primavera árabe'.

En la plataforma virtual los ciudadanos pueden publicar y proponer historias durante las 24 horas del día y cuenta como pilar fundamental con la herramienta Storify, que incluye el contenido generado en las redes sociales. Además, siguiendo con su filosofía de participación e interactividad, todas las narraciones pueden ser compartidas a través de Twitter, Facebook y Google + o recibir comentarios para generar el debate colectivo entre los usuarios de la plataforma.

Estas historias visualmente conforman un collage de visiones y aportaciones de sus propios protagonistas, ciudadanos que viven los sucesos en primera persona o usuarios muy vinculados al tema central que manifiestan su opinión o contribuyen a la información otros datos de contexto. La mayoría de aportaciones para construir la información provienen de la red social Twitter, pero también pueden encontrarse numerosos vídeos alojados en Youtube, enlaces a blogs o a otras plataformas sociales $\mathrm{y}$, en menor medida, documentos de texto. Algunas de las narraciones quedan únicamente alojadas en la plataforma, a disposición de los usuarios, pero las más destacadas e interesantes son seleccionadas para el programa televisivo. Su formato, que normalmente de aproximadamente 45 minutos de duración, es conducido por presentadores profesionales que van introduciendo y moderando la participación de los ciudadanos a través de los documentos gráficos que han aportado o con intervenciones por videoconferencia durante el programa. Por último, para completar toda esta dinámica de participación social, el programa cuenta además con plataformas en las redes sociales Google+, Twitter, Facebook, Pinterest, Instagram, en las que invita a sus seguidores a seleccionar las historias más interesantes de su web para que formen parte del programa televisivo o a seguir los debates que se generan en torno a las historias principales. Además, cuenta con un canal de Youtube, que posibilita el visionado de sus programas y ofrece a los usuarios la posibilidad de suscripción por RSS y descarga de podcast.

\section{Conclusiones}

En Internet las redes sociales, que han sido incluidas de manera progresiva dentro de las ediciones digitales de las grandes cabeceras de los medios, ya sea como herramienta de interacción y conversación con los lectores o como una forma de difusión unidireccional de los contenidos del medio, han instaurado en sus usuarios una costumbre de participación, a través de la difusión de los enlaces o la opinión sobre sus informaciones. Además, las herramientas como los blogs o las plataformas para alojar contenidos multimedia han facilitado la producción de contenidos cuya 
autoría pertenece a los ciudadanos y que tienen potencial para ser consumidos por cualquier usuario de la red. Estos factores han provocado que el denominado 'periodismo ciudadano' se multiplique como alternativa a la información de los medios oficiales, a pesar de las reiteradas críticas al peligro de la ausencia de un profesional que garantice el criterio de selección, la veracidad y el contraste de los contenidos. Los medios de comunicación se han visto obligados a captar esta tendencia y cada vez más se involucran en proyectos que incentiven la participación ciudadana aunada al trabajo de periodistas profesionales en plantilla. The Stream es un ejemplo exitoso de estas plataformas a través de la colaboración entre ciudadanos para crear historias en redes sociales, que son albergadas en la página web del programa, y el trabajo de los periodistas en la selección de la información más relevante, contrastación de la información y la elaboración del programa televisivo donde se utilizan las historias de los usuarios.

\section{Bibliografía}

ABBATE, J (2000). Inventing the Internet. Cambridge: MIT Press.

CABRERA GONZÁLEZ et al (2010). Evolución tecnológica y Cibermedios. Zamora: Comunicación social ediciones y publicaciones.

CASTELLS OLIVÁN, M (2004). La Sociedad en Red: Una visión global. Madrid: Alianza Editorial.

CEBRIÁN HERRERPS, M (2009). "Comunicación interactiva en los cibermedios." Revista En: Científica De Educomunicación, XVII(33), 15-24. Disponible en http://www.revistacomunicar.com/index.php?contenido=detalles\&numero=33\&articulo=33-2009-03. [19-07-2013]

CONTRERAS, T (2008). "La web participativa: Blogs, el periodismo ciudadano y la democracia". En: Razón y Palabra, 60 Disponible en: http://www.razonypalabra.org.mx/anteriores/n60/varia/tcontreras.htm Consultado el 20 de julio de 2013. [20-07-2013]

DANS, E (2010). Todo va a cambiar - tecnología y evaluación: Adaptarse o desaparecer. Barcelona: Deusto S.A. Ediciones.

DÍAZ NOCI, J (2006). "La interactividad y el periodismo online: Una aproximación teórica al estado de la cuestión”. En: Diálogos Possíveis, 5(2), 7-28.

EL-NAWAWY, M y ISKANDAR, A (2010). "Al-Jazeera: La revolución de los medios de información en el mundo árabe". En: Alif Nûn, 38 disponible en http:// www.libreria-mundoarabe.com/Boletines/n\%BA86\%20Oct.10/AlJazeera.htm [22-07-2013]

ESPÍRITU SANTO, N. et al (2011). Periodismo ciudadano. Evolución positiva de la comunicación. Madrid: Fundación Telefónica

GONZÁLEZ-QUIJANO, Ybes (2011). "Las revueltas árabes en tiempos de transición digital. Nueva Sociedad 235". Disponible en http://library.fes.de/pdf-files/ nuso/nuso-235.pdf [27-07-2013] 
GUIOTE, A (2008). "Entre los bastidores del Conocimiento 2.0: el perfil, las reflexiones y las rutinas de acción de los constructores del contenido en Wikipedia". En: Zer, 16(30), 13-26.

KAUSH, K (2011). "Mitos de la revolución y escenarios en Oriente Próximo". En: Política Exterior. 140, 24-31

MESO ARYEDI, K. (2005). "Periodismo Ciudadano: voces paralelas a la profesión periodística. Revista Latinoamericana de Comunicación” En: CHASQUI. 090,415

NOGUERA, J. M. y CORREYERO, B. (2008). "El periodismo ciudadano en la cobertura de la masacre de Virginia". En: Textual \& Visual Media, 1, 237-254.

PABU-TARBUSH, J (2011). "Perfiles sociopolíticos de la primavera árabe". En: actas del X Congreso de AECPA. Disponible en http://www.aecpa.es/uploads/ files/modules/congress/10/papers/249.pdf [03-08-2013]

SCOLARI, C (2008). Hipermediaciones. Elementos para una teoría de la Comunicación Digital Interactiva. Barcelona: Editorial Gedisa.

\section{La autora}

Cristina Renedo Farpón. Licenciada en Periodismo por la Universidad de Valladolid y estudiante del Máster en la Sociedad de la Información el Conocimiento de la Universitat Oberta de Catalunya. Trabaja desde 2010 en Culturatic, una empresa de comunicación digital, donde ejerce tareas de comunicación en redes sociales y plataformas digitales, periodismo web y apoyo a gabinetes de prensa en el ámbito 2.0. 